\title{
Coffee, Donuts, and Semiconductors
}

\section{During the Semiconductors for Breakfast meeting, researchers woke up to talks on light-emitting nanorods and hairy cavities.}

\author{
By Michael Schirber
}

6 "I eat semiconductors for breakfast" sounds like a line from one of the Terminator movies. But these widely used materials were on the morning menu at a seminar series hosted by the German Physical Society (DPG) last month. The Semiconductors for Breakfast series ran for two weeks and offered a wide buffet of topics from light-emitting nanorods to gas-sensing lasers. Attendees were treated each morning to a colloquium-style talk in a casual, bring-your-own-coffee virtual setting.

"The talks highlight current topics in the field of modern semiconductor physics and aim at a broad audience, including

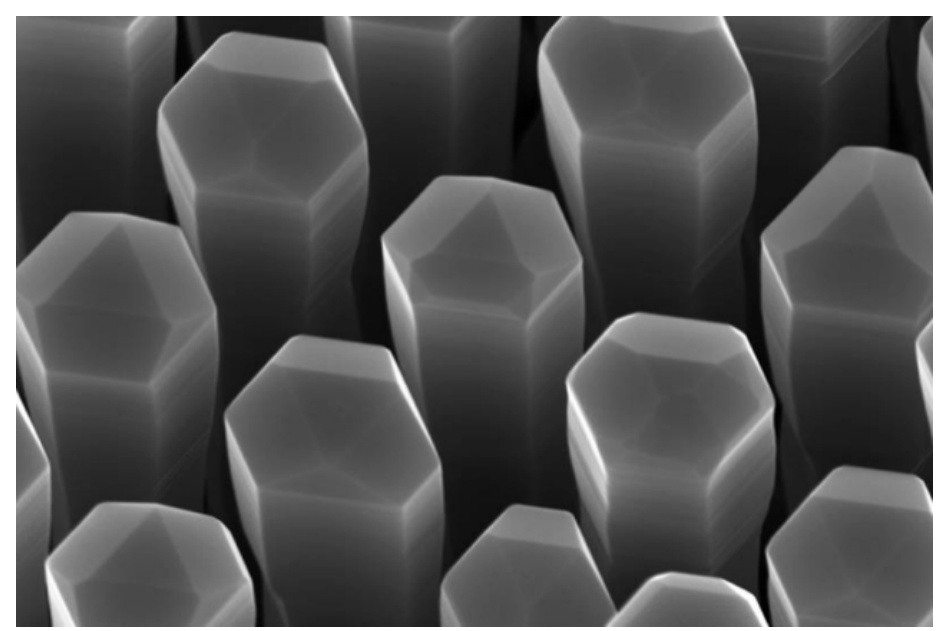

Recent work shows that nanorods of silicon-germanium can emit light, unlike pure silicon crystals. Researchers grew these semiconductor materials with the necessary hexagonal crystal structure by seeding them on hexagonally shaped nanowires. Credit: E. Fadaly/Eindhoven University of Technology students, Ph.D. students, postdocs, and senior researchers," says co-organizer Michael Lorke from the University of Bremen, Germany. The series originated last year as a virtual stand-in for the DPG Spring Meeting, which was cancelled because of the COVID-19 pandemic. This year, the online-breakfast-table format was again offered to the semiconductor community, as in-person meetings remain a problem. The turnout, like last year, was quite good, with an average of around 120 participants per talk. "I'm really glad that that so many people came back this year," says co-organizer Doris Reiter from the University of Münster in Germany.

The format of the meeting was open, without any formal registration. The only requirement was to click on the link at the appropriate morning hour. With only one talk per day, attendees could join for an extended coffee break without having to disrupt their entire work schedule. "One talk is a good amount to digest," Reiter says. "And it gives you a reason to get up in the morning!" It also provided more freedom for discussions. "We had occasions where the questions at the end of the talk went on for one and a half hours," Lorke says. "You simply cannot do that if you pack all the talks into a few days," he says.

The sessions kicked off with a presentation on hexagonal silicon-germanium crystals by Erik Bakkers from Eindhoven University of Technology in the Netherlands. This new light-emitting semiconductor was selected as the breakthrough of the year by Physics World magazine in 2020. Pure silicon crystals can't emit light because they have an indirect band gap, which means the conduction electrons have a momentum offset with respect to the ground state. To create a direct band 
gap in silicon-germanium, Bakkers and his colleagues had to force the material into a hexagonal lattice structure, which they did by growing silicon-germanium nanorods around a hexagonal crystal "seed." The nanorods emit light at infrared wavelengths-making them a promising candidate for converting electric signals into photon signals on computer chips.

Other talks ran the gamut of semiconductor physics, from lasers to quantum dots to brain-inspired memory devices. Jesper Mørk from the Technical University of Denmark presented some recent work on photonic crystals, which are fabricated periodic structures that can manipulate light. Mørk's group has developed photonic crystals that combine a waveguide with a small cavity. Such devices can perform as optical switches, as sensors, and, as demonstrated more recently, as lasers. To boost the performance of these photonic crystals, the team is working on reducing the size of the cavity, which should strengthen the coupling of light to other objects, such as single-atom switches. Numerical calculations by the group have uncovered volume-minimizing cavity shapes that achieve this performance enhancement. Mørk shared some images of these topology-optimized cavities, which have a bow-tie center surrounded by surprisingly "hairy" structures.

Some of the speakers came from industry, like Johannes Koeth who works for nanoplus Nanosystems and Technologies, a German company that supplies a wide range of tunable diode lasers for sensing applications. These sensors can target specific absorption lines of a desired molecule. For example, a characteristic absorption feature for water vapor occurs at 1392 $\mathrm{nm}$. In a typical setup, the laser rapidly sweeps over a small range of wavelengths around this spectral feature while a detector extracts the gas concentration from the level of laser light absorption. Applications include sensing leaks from pipelines, monitoring the respiration of premature babies, and spotting a drunk driver by shining a laser through a car window to detect traces of alcohol in the driver's breath.
These and other interesting talks provided a stimulating brew, but both Lorke and Reiter are looking forward to getting back to in-person meetings. "It's vital that young people have a place to present themselves and their work," Reiter says. For now, the DPG is planning a virtual semiconductor meeting with student contributions for September 2021. But could Semiconductors

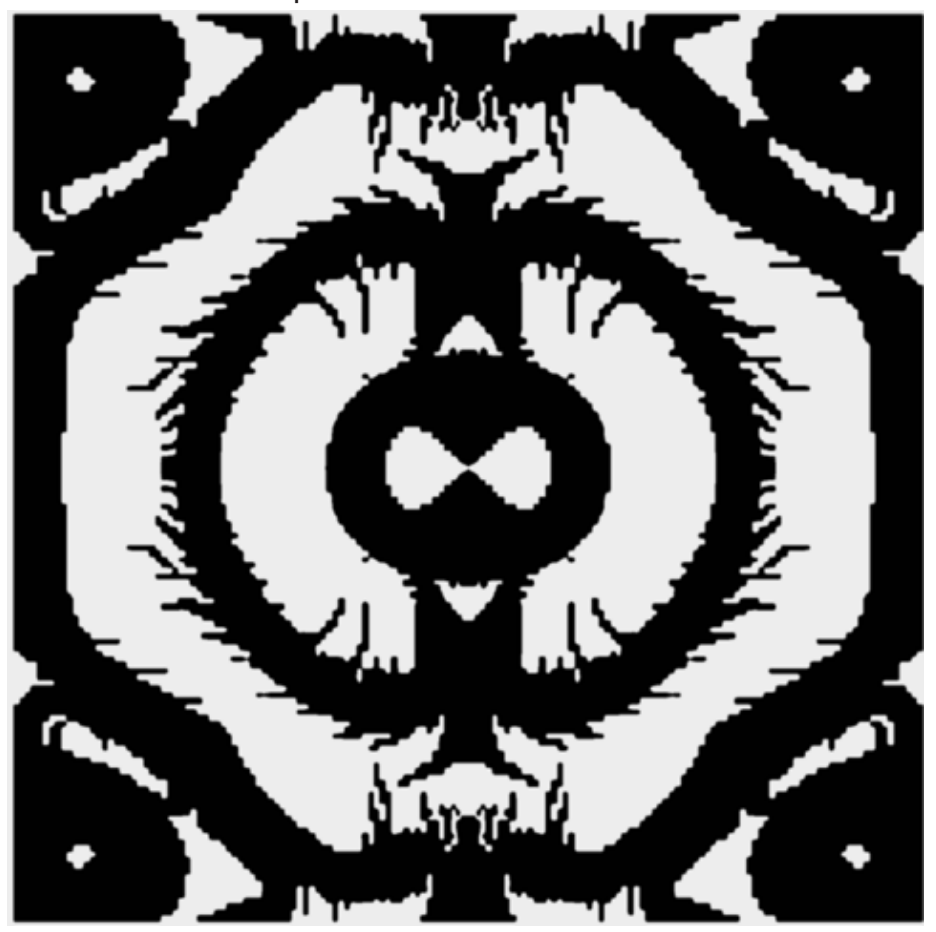

This computer drawing shows a cavity design that minimizes the volume where light is confined.

Credit: Appl. Phys. Lett. 113, 241101 (2018)/AIP Publishing

for Breakfast continue in some form? "If there is the opportunity to do it again, I would definitely jump in," Reiter says.

Michael Schirber is a Corresponding Editor for Physics based in Lyon, France. 